Check for updates

Cite this: Chem. Sci., 2019, 10, 701

๑ All publication charges for this article have been paid for by the Royal Society of Chemistry

Received 3rd October 2018

Accepted 24th October 2018

DOI: $10.1039 / \mathrm{c} 8 \mathrm{sc} 04394 a$

rsc.li/chemical-science

\section{Click to enter: activation of oligo-arginine cell- penetrating peptides by bioorthogonal tetrazine ligations $\uparrow$}

\author{
Saskia A. Bode, $\$$ Suzanne B. P. E. Timmermans, (D) Selma Eising, \\ Sander P. W. van Gemert, Kimberly M. Bonger (D) * and Dennis W. P. M. Löwik (D)*
}

Cell-penetrating peptides are able to transport a wide variety of cargo across cell membranes. Although promising, they are not often considered for therapeutic purposes as they lack controllable activity and cell selectivity. We have developed an activation strategy based on a split octa-arginine cell-penetrating peptide (CPP) that can be activated by means of bioorthogonal ligation. To this end we prepared two non-penetrating tetra-arginine halves, functionalized either with a tetrazine or with a complementary bicyclo[6.1.0]nonyne (BCN) group. We demonstrate that an active octa-arginine can be reconstituted in situ upon mixing the complementary split peptides. The resulting activated peptide is taken up as efficiently as the well-established cell-penetrating peptide octa-arginine. The activation of the oligoarginines can also be achieved using trans-cyclooctene (TCO) as a ligation partner, while norbornene appears too kinetically slow for use in situ. We further show that this strategy can be applied successfully to transport a large protein into living cells. Our results validate a promising first step in achieving control over cell penetration and to use CPPs for therapeutic approaches.

\section{Introduction}

Cell-penetrating peptides (CPPs) have the ability to penetrate the otherwise impermeable plasma membrane. They can transport large cargoes and as a result are potential candidates for delivery of bioactive molecules..$^{1-5}$ Although the use of CPPcargo constructs for cellular delivery is promising, CPPs are hardly cell-type specific, and there is no temporal control over their uptake..$^{6-8}$ The use of CPPs for therapeutic purposes would greatly benefit if cell-type selectivity and control over their moment of uptake could be induced. ${ }^{9}$ Various studies have been reported on the development of activatable CPPs, using several triggers, such as a change in $\mathrm{pH},{ }^{10}$ irradiation, ${ }^{11-13}$ or enzymatic reactions. ${ }^{14,15}$ Most of these activation strategies promise to induce cellular uptake. They generally depend on the properties of the targeted tissue or cell type, making them sensitive for changes in the targeted environment. Now, we think it would be beneficial to develop an activation strategy that does not need an enzymatic activity or $\mathrm{pH}$ change. When we would combine it with a targeting strategy it can rely on any receptor or other type of marker and therefore would be more generally applicable.

Institute for Molecules and Materials, Radboud University, Heyendaalseweg 135, 6525 AJ Nijmegen, The Netherlands. E-mail: dennis.lowik@ru.nl; k.bonger@science.ru.nl $\dagger$ Electronic supplementary information (ESI) available. See DOI: 10.1039/c8sc04394a

\$ These authors contributed equally.
Previously, we demonstrated an activation strategy for oligoarginines. ${ }^{16,17}$ For these peptides it is known that a minimum amount of six arginine residues is needed to induce cellular uptake. $^{18,19}$ Making use of this characteristic, a split-CPP approach was designed that involved truncated oligo-arginines consisting of three to five arginine residues and a C-terminal cysteine. These peptides were assembled and activated through disulfide bridge formation with complementary oligoarginines containing an $\mathrm{N}$-terminal cysteine residue. Whereas the truncated split peptides did not induce uptake in HeLa cells, the assembled octa- and nona-arginines were efficient CPPs. Although we demonstrated that activation of short oligoarginine peptides is possible by extension of their sequence, its dependency on the formation of disulfide bridges hampers their use for in situ activation due to the dominant oxidative environment and the presence of potentially interfering thiols in many cellular environments. To overcome this limitation, and to circumvent possible non-specific interactions of the split CPPs with endogenous biomolecules, we investigated alternative activation strategies based on the bioorthogonal tetrazine ligation (Fig. 1). ${ }^{20}$

\section{Results and discussion}

\section{Design and synthesis}

The tetrazine ligation with alkenes or alkynes is based on an inverse electron demand Diels-Alder (iEDDA) reaction and has been used previously for several in vivo applications. ${ }^{21-26}$ For our 


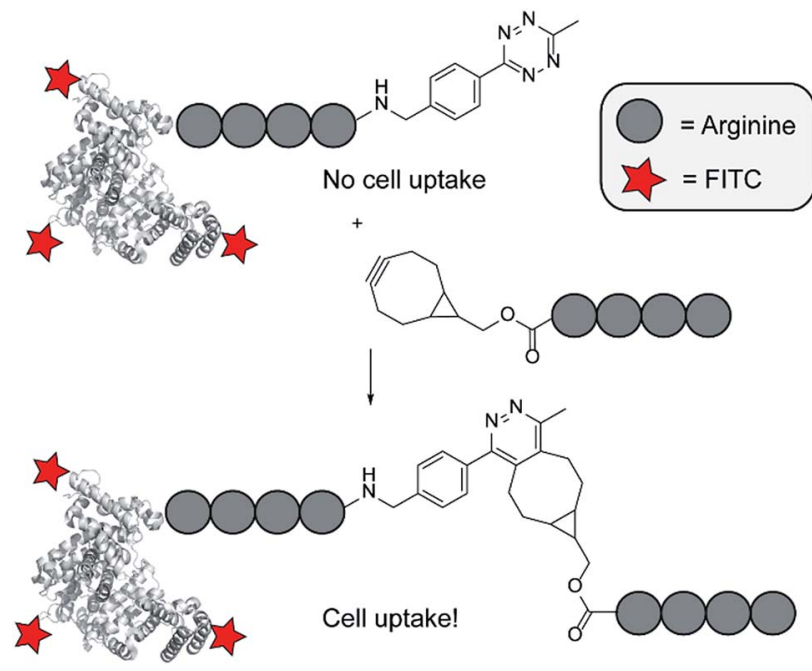

Fig. 1 Overview of the click to enter strategy.

in situ activation strategy of CPPs we synthesized several truncated tetra-arginines equipped with a tetrazine moiety or with a complementary alkene and alkyne reactive group. We first prepared a tetra-arginine, of which the C-terminus was connected to a 3-aminobenzyl-6-methyl substituted tetrazine ${ }^{27}$ (R4-Tz, Fig. 2). We selected this tetrazine, because of its stability and solubility in aqueous environments. Although the reactivity of this tetrazine is moderate compared to other more electrondeficient less stable tetrazines, the reaction rates are still suitable for biological applications. ${ }^{28}$ Further, we labelled the $\mathrm{N}$-terminus with fluorescein isothiocyanate (FITC), linked via

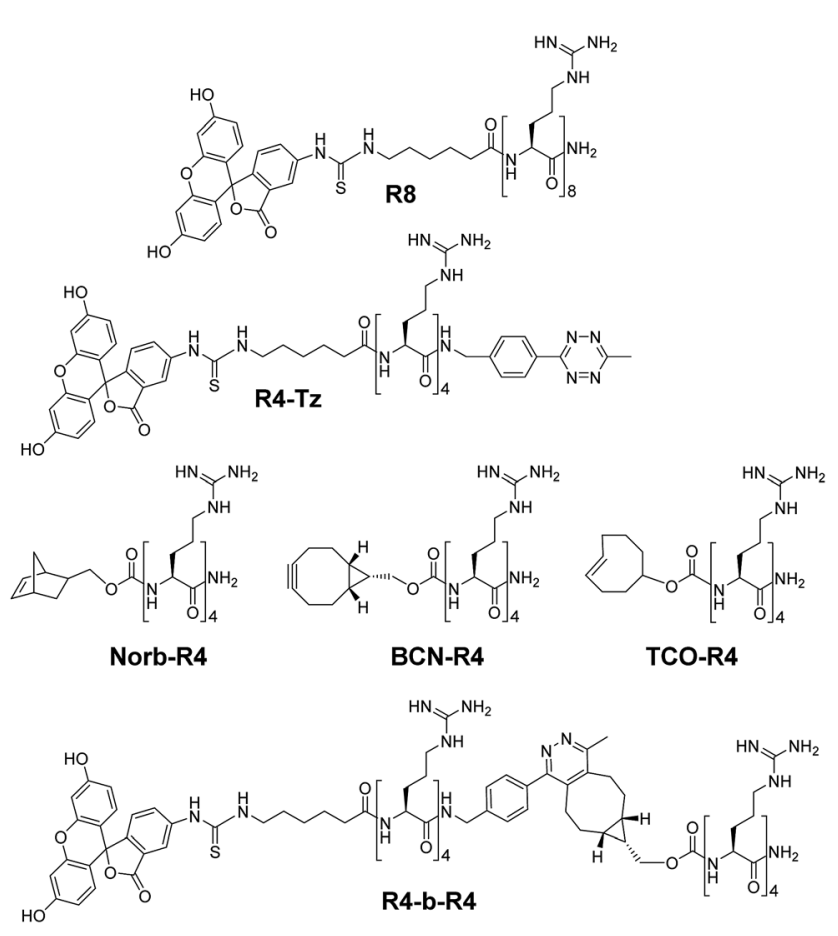

Fig. 2 Structures of oligo-arginine peptides used in this study. a 6-aminohexanoic acid spacer, to enable the visualization of cellular uptake with confocal laser scanning microscopy (CLSM) and quantification using flow cytometry. As an activation partner for the R4-Tz we first explored a tetra-arginine containing the bioorthogonal bicyclo[6.1.0]nonyne (BCN-R4)..$^{25,29}$ In addition, we synthesized fluorescently labelled octa-arginine $\mathbf{R 8}$ as a reference CPP and pre-conjugated R4-b-R4 - prepared from R4-Tz and BCN-R4 - that allowed us to determine the activity of the fully reconstituted CPP and find suitable conditions for uptake (Fig. 2).

\section{Uptake of pre-ligated conjugates}

The mechanism of uptake of arginine-rich CPPs is dependent on their concentration and their structures, ${ }^{30}$ therefore we started by comparing the uptake of CPP R8 with that of the pre-ligated peptide R4-b-R4 at $5 \mu \mathrm{M}, 10 \mu \mathrm{M}, 15 \mu \mathrm{M}$ and $20 \mu \mathrm{M}$ using confocal fluorescence microscopy (Fig. 3). As a control, we measured the uptake of the truncated peptide R4-Tz. At low concentrations ( $5 \mu \mathrm{M}$ and $10 \mu \mathrm{M})$, we did not observe any uptake of the truncated peptide R4-Tz, which was in accordance with earlier findings that demonstrated that at least six arginines within one sequence are required for efficient cellular uptake. ${ }^{16}$ At higher concentrations ( $15 \mu \mathrm{M}$ and $20 \mu \mathrm{M})$ we observed some
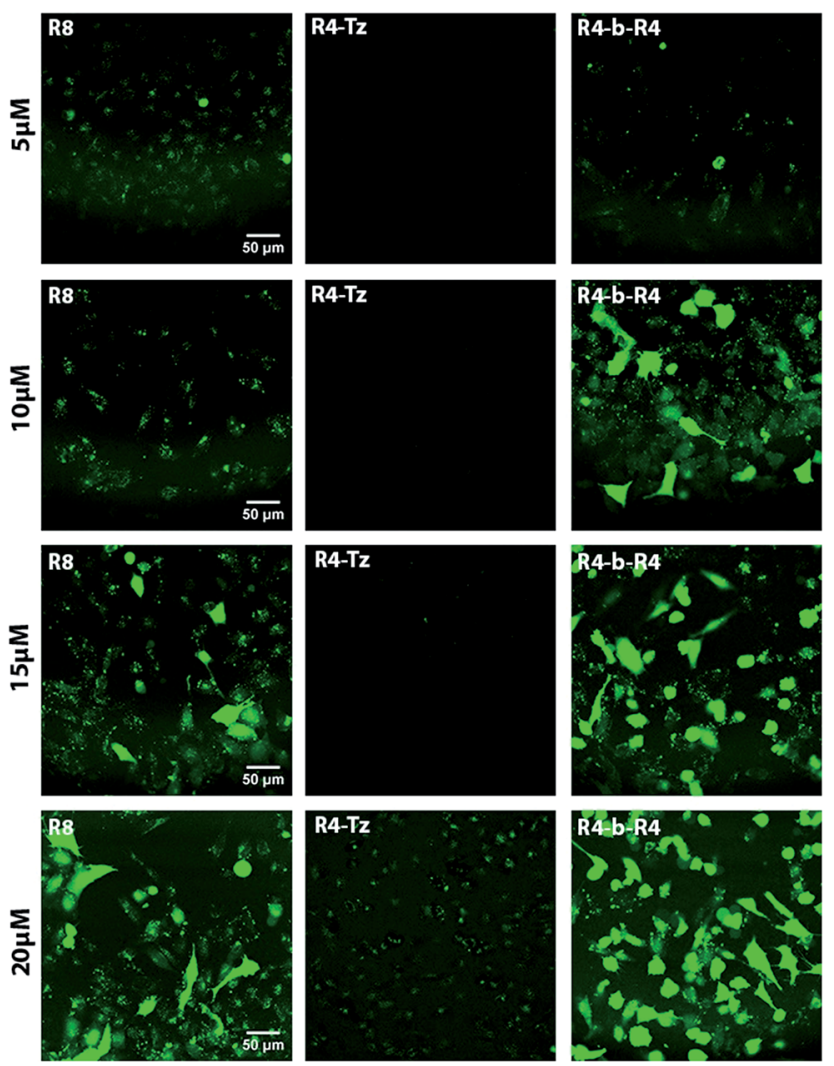

Fig. 3 CLSM micrographs corresponding to cellular uptake studies in HeLa cells. The concentration of the CPP increases from top to bottom $(5,10,15$ and $20 \mu \mathrm{M})$. The experiments were performed with reference peptide $\mathrm{R} 8$, pre-ligated $\mathrm{R} 4-\mathrm{b}-\mathrm{R} 4$ and truncated $\mathrm{R} 4-\mathrm{Tz}$ at $37{ }^{\circ} \mathrm{C}$, with an incubation time of 30 minutes in serum-containing medium. 
minor cellular internalization. The pre-ligated peptide $\mathbf{R 4 - b - R 4}$ and $\mathbf{R 8}$ were taken up at all concentrations, however, the mechanism of uptake varied considerably between the peptide concentrations (Fig. 3). At low concentrations, R8 and R4-b-R4 were taken up most likely via endocytosis, suggested by the punctuate fluorescence in the cells, while strong cytosolic uptake was observed at the higher concentrations, possibly via nucleation zones as has been reported before by $\mathrm{us}^{\mathbf{1 6}}$ and for arginine rich CPPs in general. ${ }^{31}$ The concentration dependent uptake of arginine-rich CPPs has been described in the literature before, however, it was surprising that the concentration at which HeLa cells switched to a different uptake mechanism differed between the two similar peptides R8 and R4-b-R4. From these results and for uniformity, we concluded that $5 \mu \mathrm{M}$ would be the best concentration to compare in situ activation for these small molecules.

\section{In situ activation}

The efficient cellular uptake of the $\mathbf{R 4 - b}-\mathbf{R 4}$ peptide, prompted us to study the in situ activation of oligo-arginines using the bioorthogonal tetrazine ligation. We commenced by co-incubating HeLa cells with $5 \mu \mathrm{M}$ R4-Tz in serum-containing culture medium and added 5 eq. of BCN-R4 to allow ligation and formation of $\mathbf{R 4 - b}-\mathbf{R 4}$. We included $\mathbf{R 8}$ as positive control, while R4-Tz served as negative control. As seen by CLSM, the uptake of in situ activated R4-Tz $+\mathbf{B C N}-\mathbf{R 4}$ at 30 minutes was similar to the uptake of $\mathbf{R 8}$ as well as $\mathbf{R 4 - b}-\mathbf{R 4}$, indicating that in situ activation of R4-Tz to R4-b-R4 is possible at these conditions (Fig. 4 and $\mathrm{S} 1 \dagger$ ).

Next, we evaluated whether uptake could be improved by increasing the amount of activating agent BCN-R4 or by prolonging the incubation time. We found that addition of 10 eq. of BCN-R4 instead of 5 eq. did not improve uptake (Fig S2 $\dagger$ ), while reducing the amount of $\mathbf{B C N}-\mathbf{R 4}$ from 5 eq. to 2.5 eq. resulted in a significant decrease in cellular uptake (Fig. S3†). Subsequently, we assessed the effect of incubation time on in situ activation of R4-Tz. Using similar conditions as above we observed CPP uptake already after 30 minutes and that uptake increased slightly over time. At 60 minutes, similar uptake levels were reached as observed after 90 minutes (Fig. S3†). From these results we concluded that 30-60 minutes of incubation using 5 eq. of BCN-R4 activating agent are needed for sufficient CPP uptake.

To study the effect of the tetrazine ligation rate, we additionally prepared tetra-arginine fragments that were $\mathrm{N}$-terminally equipped with either a norbornene (Norb-R4), or a trans-cyclooctene (TCO-R4) moiety (Fig. 2). Norbornene and TCO are known to display a respectively lower and higher reactivity towards tetrazines compared to $\mathrm{BCN} .{ }^{25}$ We compared the uptake of the in situ activation of R4-Tz by the tetra-arginines with the different bioorthogonal reagents using CLMS (Fig. 4A). To acquire the uptake efficiency more quantitatively, we additionally measured the cellular fluorescence of the various tetrazine reactions with flow cytometry (Fig. 4B). HeLa cells were incubated with R8 at $5 \mu \mathrm{M}$ for 30 minutes at $37^{\circ} \mathrm{C}$ in serum containing medium and compared to cells that were incubated for 30 minutes with $5 \mu \mathrm{M} \mathrm{R4-Tz}$ to

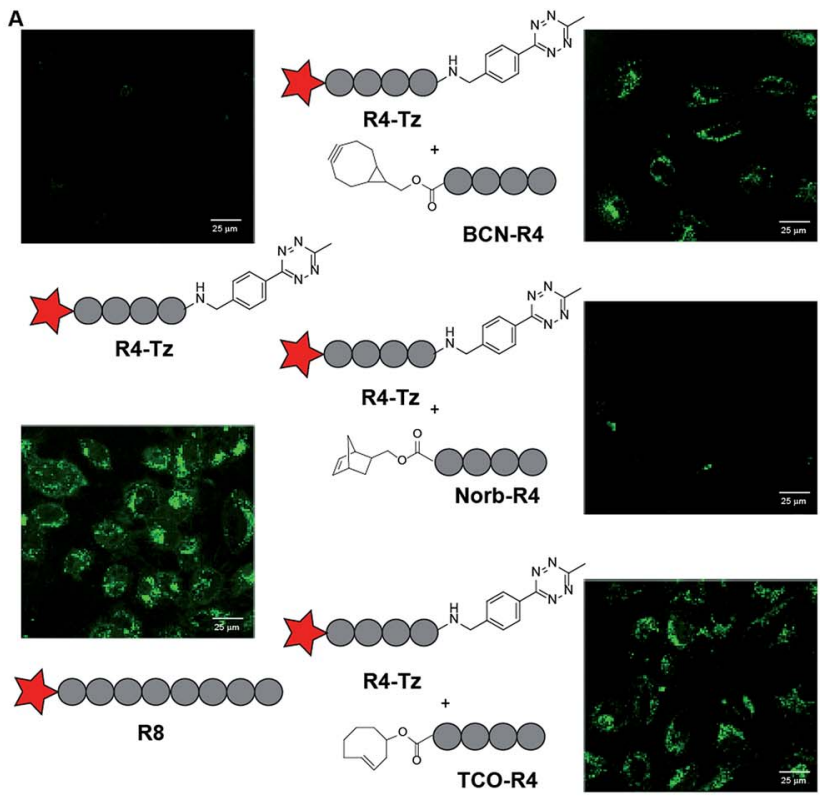

B

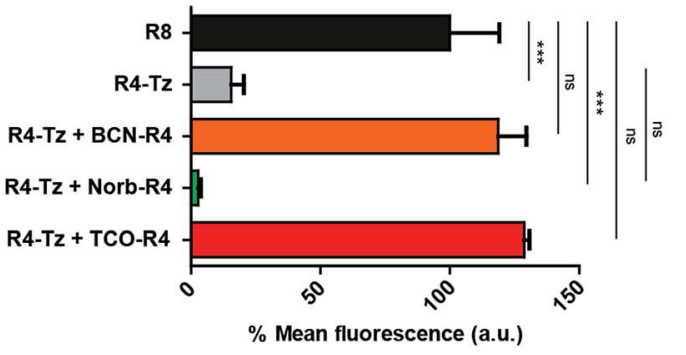

Fig. 4 HeLa cell uptake studies to test in situ activation. (A) CLSM micrographs for HeLa cell uptake studies to test in situ activation. CSLM images are depicted for positive control R8, negative control R4-Tz and in situ activated R4-Tz + BCN-R4/Norb-R4/TCO-R4. For the in situ activation HeLa cells were incubated with $5 \mu \mathrm{M}$ R4-Tz and 5 eq. of BCN-R4/Norb-R4/TCO-R4 for 30 minutes. (B) Flow cytometry results at $5 \mu \mathrm{M}$ in serum-containing medium for 30 minutes of incubation.

which 5 equivalents of BCN-R4, Norb-R4, or TCO-R4 were added respectively. As shown in Fig. 4, truncated split R4-Tz again hardly gave any uptake. Interestingly, the R4-Tz peptide incubated with Norb-R4 showed only poor uptake while both activation with BCN-R4 and TCO-R4 gave uptake that was comparable to reference peptide R8. Interestingly, no significant difference was found between BCN and TCO.

\section{Protein cargo}

As our activation strategy is feasible using an iEDDA conjugation reaction for a small molecule fluorophore we next explored whether our strategy could also be used for the induced uptake of a much larger protein cargo. To this end we selected fluorescently labelled human serum albumin (HSA) as a model protein. This 66 $\mathrm{kDa}$ protein contains a single free cysteine that allowed us to site selectively modify the protein with a tetrazine. This was achieved by first reacting the cysteine of HSA with a bi-functional maleimide-DBCO ${ }^{32}$ linker and reaction of free lysines of HSA with 
A

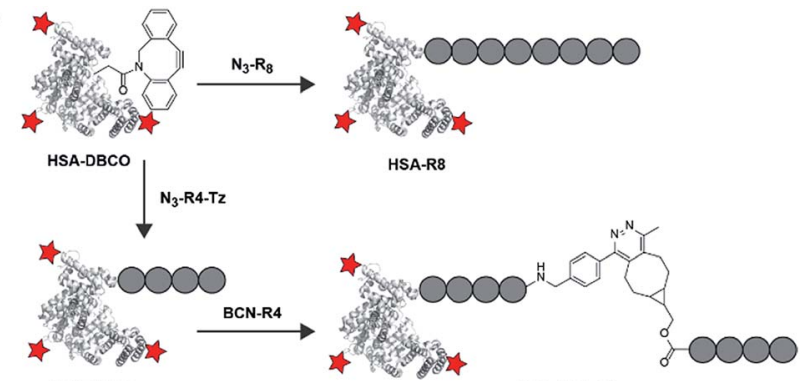

HSA-R4-Tz

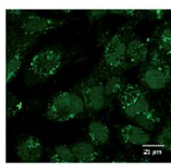

HSA-DBCO

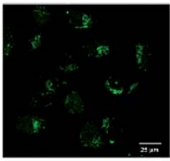

HSA-R4-Tz

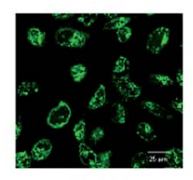
HSA-R8

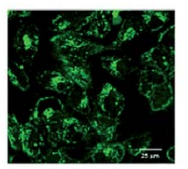

HSA-R4-b-R4

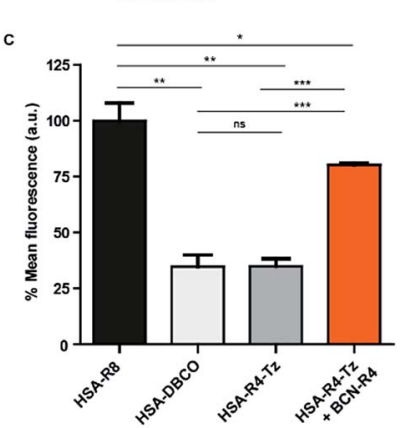

Fig. 5 HSA conjugates and HeLa cell uptake studies to test in situ activation. (A) Structures of the molecules used to modify HSA. (B) Confocal fluorescence images at $10 \mu \mathrm{M}$ in serum-containing medium for 90 minutes of incubation. (C) Flow cytometry results under the same conditions.

FITC providing fluorescent HSA-DBCO. A tetra-arginine with an N-terminal azide and a C-terminal tetrazine, N3-R4-Tz, was conjugated in a sequential step yielding HSA-R4-Tz (Fig. 5A). In addition, we prepared an octa-arginine protein conjugate (HSA-R8) using N3-R8, for comparison.

Using HSA as cargo conjugated to a tetrazine oligo-arginine, activation with the BCN-R4 peptide under the conditions employed above, we did not observe significant cellular uptake as evidenced by FACS as well as confocal microscopy. Fortunately, incubation at a slightly higher concentration $(10 \mu \mathrm{M})$ and increased time (90 min) resulted in clear uptake of the HSAR4-Tz protein which was in situ activated by conjugation to BCNR4 (Fig. 5B). Similar uptake levels were observed for HSA-R8 while unreacted HSA-R4-Tz was only poorly taken up. These qualitative results were quantified using flow cytometry using the same conditions (Fig. 5C) and substantiate that our in situ CPP activation strategy is suitable for the delivery of a large protein cargo.

\section{Conclusions}

In conclusion, we have successfully developed an in situ activation strategy for oligo-arginine CPPs based on a bioorthogonal tetrazine ligation between two split tetra-arginine parts. The norbornene-based reaction did not show kinetics suitable for in situ application, but both BCN and TCO were efficient in reconstituting active CPPs. This provides a valuable method for probe design and is the basis for further efforts in this line of research. Moreover, using BCN efficient cellular

uptake was observed for a low molecular weight fluorescent probe as well as a large protein cargo. Future efforts in this line of research are to incorporate homing peptides or targeting ligand $\mathrm{s}^{33}$ in our tetra-arginine activation strategy, providing the first steps towards a targeted and site-selective cargo delivery strategy involving activatable CPPs.

\section{Conflicts of interest}

There are no conflicts to declare.

\section{Acknowledgements}

The department of General Instruments of the Radboud University, and Elisabeth Pierson in particular, is acknowledged for providing microscopy services.

\section{Notes and references}

1 D. Zhang, J. Wang and D. Xu, J. Controlled Release, 2016, 229, 130-139.

2 F. Wang, Y. Wang, X. Zhang, W. Zhang, S. Guo and F. Jin, J. Controlled Release, 2014, 174, 126-136.

3 K. Kurrikoff, M. Gestin and Ü. Langel, Expert Opin. Drug Delivery, 2016, 13, 373-387.

4 A. Komin, L. M. Russell, K. A. Hristova and P. C. Searson, Adv. Drug Delivery Rev., 2017, 110-111, 52-64.

5 L. Feni and I. Neundorf, The Current Role of Cell-Penetrating Peptides in Cancer Therapy, in Peptides and Peptide-based Biomaterials and their Biomedical Applications, Advances in Experimental Medicine and Biology, ed. A. Sunna, A. Care and P. Bergquist, Springer, Cham, 2017, vol. 1030, pp. 279295.

6 Y. Huang, Y. Jiang, H. Wang, J. Wang, M. C. Shin, Y. Byun, H. He, Y. Liang and V. C. Yang, Adv. Drug Delivery Rev., 2013, 65, 1299-1315.

7 Q. Zhang, H. Gao and Q. He, Mol. Pharm., 2015, 12, 31053118.

8 E. Vives, J. Controlled Release, 2005, 109, 77-85.

9 S. R. MacEwan and A. Chilkoti, Wiley Interdiscip. Rev.: Nanomed. Nanobiotechnol., 2013, 5, 31-48.

10 E. Jin, B. Zhang, X. Sun, Z. Zhou, X. Ma, Q. Sun, J. Tang, Y. Shen, E. Van Kirk, W. J. Murdoch and M. Radosz, J. Am. Chem. Soc., 2013, 135, 933-940.

11 M. B. Hansen, E. van Gaal, I. Minten, G. Storm, J. C. M. van Hest and D. W. P. M. Löwik, J. Controlled Release, 2012, 164, 87-94.

12 J. Wang, S. Shen, D. Li, C. Zhan, Y. Yuan and X. Yang, Adv. Funct. Mater., 2018, 28, 1704806.

13 A. Prestel and H. M. Moller, Chem. Commun., 2016, 52, 701704.

14 T. Jiang, E. S. Olson, Q. T. Nguyen, M. Roy, P. A. Jennings and R. Y. Tsien, Proc. Natl. Acad. Sci. U. S. A., 2004, 101, 1786717872.

15 S. A. Bode, M. B. Hansen, R. A. J. F. Oerlemans, J. C. M. van Hest and D. W. P. M. Löwik, Bioconjugate Chem., 2015, 26, 850-856. 
16 S. A. Bode, R. Wallbrecher, R. Brock, J. C. M. van Hest and D. W. P. M. Löwik, Chem. Commun., 2014, 50, 415-417.

17 S. A. Bode, I. C. Kruis, H. P. J. H. M. Adams, W. C. Boelens, G. J. M. Pruijn, J. C. M. van Hest and D. W. P. M. Löwik, ChemBioChem, 2017, 18, 185-188.

18 S. Futaki, T. Suzuki, W. Ohashi, T. Yagami, S. Tanaka, K. Ueda and Y. Sugiura, J. Biol. Chem., 2001, 276, 5836-5840.

19 E. G. Stanzl, B. M. Trantow, J. R. Vargas and P. A. Wender, Acc. Chem. Res., 2013, 46, 2944-2954.

20 D. M. Patterson and J. A. Prescher, Curr. Opin. Chem. Biol., 2015, 28, 141-149.

21 J. Sauer, D. K. Heldmann, J. Hetzenegger, J. Krauthan, H. Sichert and J. Schuster, Eur. J. Org. Chem., 1998, 2885-2896. 22 R. Selvaraj and J. M. Fox, Curr. Opin. Chem. Biol., 2013, 17, 753-760.

23 J. Seckute and N. K. Devaraj, Curr. Opin. Chem. Biol., 2013, 17, 761-767.

24 K. Lang and S. Mayer, Synthesis, 2016, 49, 830-848.
25 B. L. Oliveira, Z. Guo and G. J. L. Bernardes, Chem. Soc. Rev., 2017, 46, 4895-4950.

26 R. Rossin, R. M. Versteegen, J. Wu, A. Khasanov, H. J. Wessels, E. J. Steenbergen, W. Ten Hoeve, H. M. Janssen, A. van Onzen, P. J. Hudson and M. S. Robillard, Nat. Commun., 2018, 9, 1484.

27 M. R. Karver, R. Weissleder and S. A. Hilderbrand, Bioconjugate Chem., 2011, 22, 2263-2270.

28 A. C. Knall and C. Slugovc, Chem. Soc. Rev., 2013, 42, 5131-5142.

29 J. Dommerholt, S. Schmidt, R. Temming, L. J. A. Hendriks, F. P. J. T. Rutjes, J. C. M. van Hest, D. J. Lefeber, P. Friedl and F. L. van Delft, Angew. Chem., Int. Ed., 2010, 49, 9422-9425.

30 R. Brock, Bioconjugate Chem., 2014, 25, 863-868.

31 T. Takeuchi and S. Futaki, Chem. Pharm. Bull., 2016, 64, 1431-1437.

32 A. Kuzmin, A. Poloukhtine, M. A. Wolfert and V. V. Popik, Bioconjugate Chem., 2010, 21, 2076-2085.

33 M. Wang, D. Svatunek, K. Rohlfing, Y. Liu, H. Wang, B. Giglio, H. Yuan, Z. Wu, Z. Li and J. Fox, Theranostics, 2016, 6, 887-895. 